\title{
Idiopathic spontaneous adrenal haemorrhage in pregnancy
}

\section{Introduction}

Adrenal haemorrhage is rare, with an incidence of 1 in 200000. It may present with non-specific symptoms, with the diagnosis often being made incidentally on imaging as part of investigation for other underlying pathologies.

\section{Case Report}

We present a case of a 24 year old woman who presented to the maternity assessment unit in September 2016 at 33 weeks gestation. She reported symptoms of severe left sided abdominal pain and orthopnoea. In particular, she was noted to be hypoxic on pulse oximetry when lying flat.

She was otherwise fit and well with no past medical history of note. There was no clinical evidence of cortisol, catecholamine or androgen excess. She was normotensive.

A ventilation-perfusion scan was performed in view of the aforementioned symptoms. This demonstrated two segmental mismatched defects consistent with bilateral pulmonary embolism. She was commenced on anticoagulation with treatment dose low molecular weight heparin.

\section{Imaging}

As part of investigations into the cause of her abdominal pain, an MRI abdomen was performed.

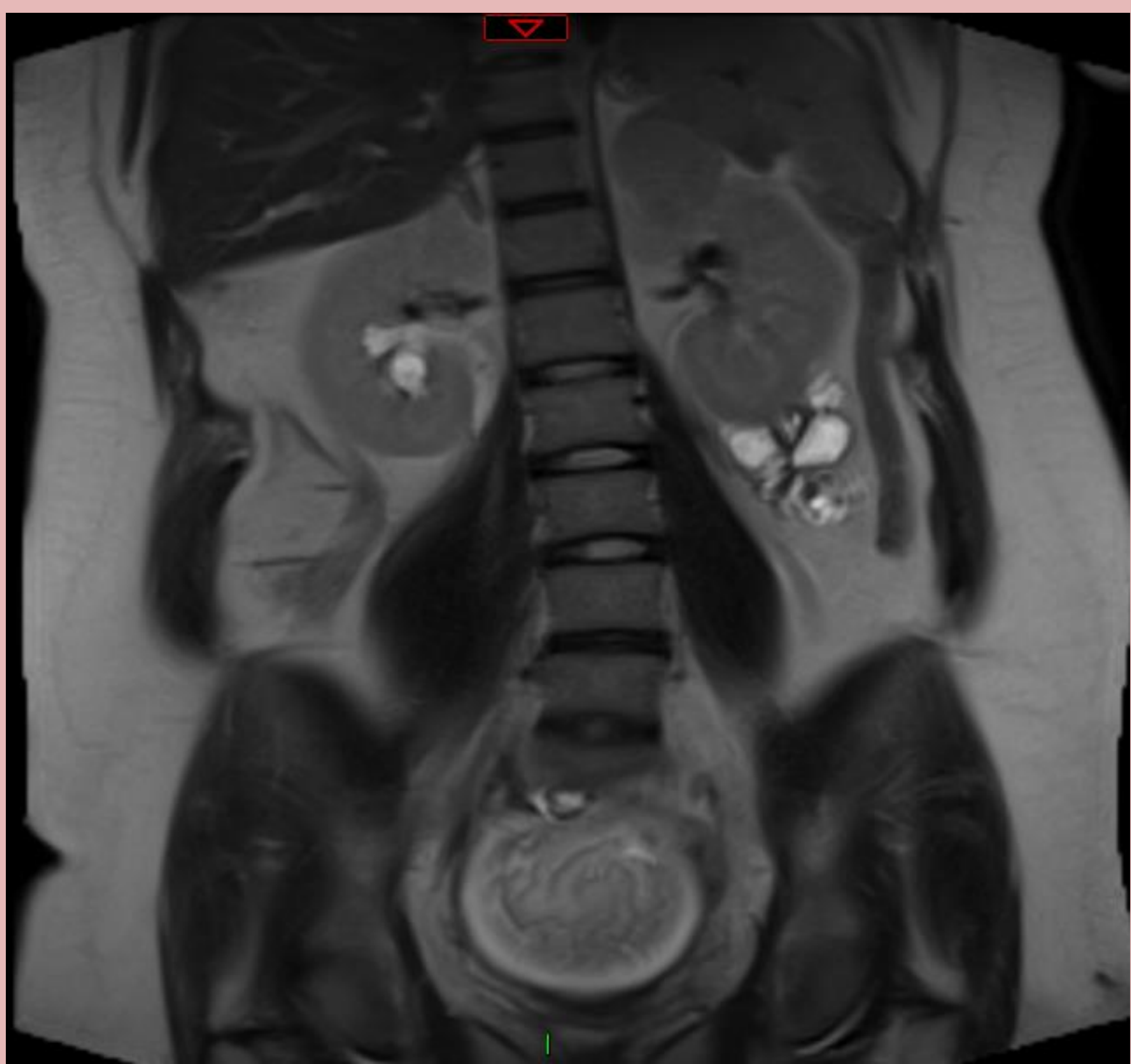

Figure 1: MRI Abdomen. There is a $4.5 \mathrm{~cm}$ left adrenal mass containing a fluid level. The appearances are suggestive of recent adrenal haemorrhage. In association with this, there is some low T2 signal which could represent blood breakdown products or calcification.

Importantly, both the ventilation-perfusion scan and the MRI abdomen were performed on the same day. The patient therefore had not been on prolonged anticoagulation prior to the detection of adrenal haemorrhage. Biochemical assessment of adrenal function was subsequently undertaken.

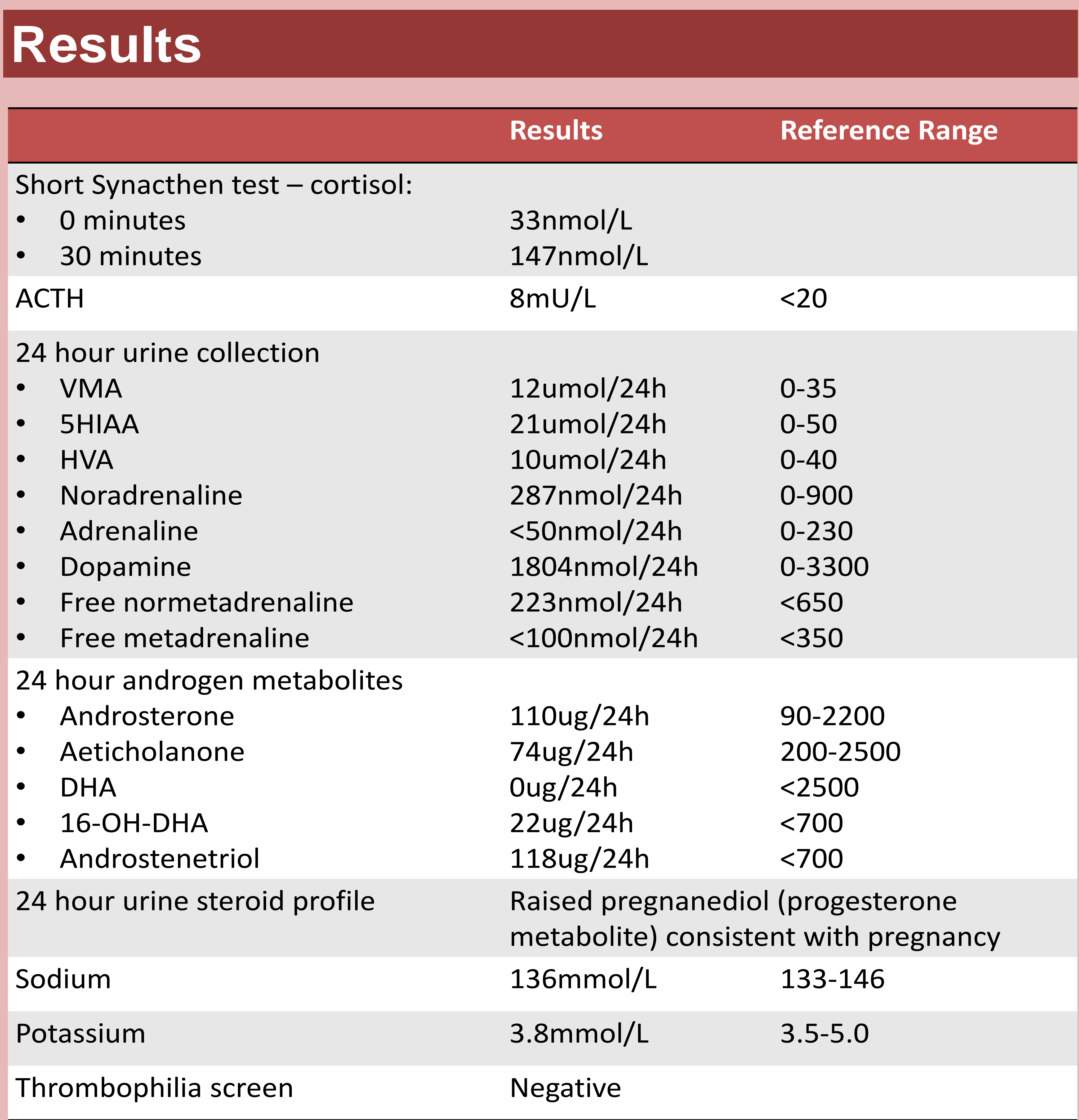

\section{Clinical Progress}

A diagnosis of left adrenal haemorrhage was made. Anticoagulation with low molecular weight heparin was continued in view of the presence of bilateral pulmonary emboli.

The blunted SST was felt to be as a consequence of administration of high dose betamethasone which had been given due to the potential for early fetal delivery, rather than true hypoadrenalism.

The remainder of her pregnancy was uneventful and she delivered at 40 weeks gestation. Post partum MRI showed a significant reduction in the size of the left adrenal mass, which now measured $1.1 \times 1.8 \mathrm{~cm}$. There were high $\mathrm{T} 1$ and $\mathrm{T} 2$ signals reflecting blood breakdown products. She was therefore likely to have undergone primary adrenal haemorrhage rather than haemorrhage into a underlying adenoma. Repeat adrenal functional testing has since been carried out and noted to be normal.

\section{Discussion}

Adrenal haemorrhage may be either unilateral or bilateral. Those with unilateral haemorrhage often do not exhibit clinical features of adrenal insufficiency and are diagnosed incidentally on imaging. Bilateral haemorrhage however commonly leads to acute adrenal insufficiency and adrenal crisis if not recognised and treated promptly.

Adrenal haemorrhage is commonly associated with meningococcal septicaemia - clasically known as Waterhouse-Friderichsen syndrome, but may also occur as a result of sepsis leading to disseminated intravascular coagulation, trauma, malignancy, anticoagulant therapy and pregnancy.

This illustrates a rare case of spontaneous adrenal haemorrhage in pregnancy. Adrenal cortex hyperplasia and hypertrophy associated with pregnancy, is thought to predispose the gland to venous congestion resulting in adrenal haemorrhage.

This case highlights the importance of considering this as a potential diagnosis in a pregnant woman who presents with abdominal pain. 